\title{
Advances in continuum modelling of urban wall structures impact
}

\author{
D. Pfau, E. L. Baker, D. Suarez, J. M. Pincay, T. Madsen \\ \& K. W. Ng \\ U.S. Army Armament Research, Development and Engineering Center, \\ Picatinny, USA
}

\begin{abstract}
Recent advancements of continuum modelling are now allowing significant improvements of urban wall structures impact simulation. Urban wall structural details can now be included in impact modelling that were not previously achievable. These modelling improvements incorporate the inclusion of resolved reinforcement bars in concrete walls, brick and mortar construction details in brick wall construction and resolved cavity and mortar configurations in brick over block wall constructions. These modelling improvements are being achieved not only due to increases in computer hardware speeds and memory, but more so due to a variety of mesh reduction methodologies that have proven to be improved modelling techniques. An adaptive mesh refinement (AMR) technique has been implemented for use in the CTH high rate Eulerian finite difference model. We have applied this AMR to successfully resolve complex material geometries and dominating physical phenomena. In addition, relaxation algorithms have been successfully applied for high rate continuum Arbitrary Lagrangian-Eulerian (ALE) modelling. These relaxation algorithms are now being used in the ALE-3D computer program to provide high resolution simulation of urban wall impacts and penetrations. These new techniques are now allowing the high rate continuum modelling of physical phenomena that was not previously simulated.
\end{abstract}

Keywords: mesh reduction methodologies, high rate continuum modelling, impact physics, high explosives. 


\section{Introduction}

High rate continuum modelling is used for the modelling of high rate events including the high velocity impact of urban structures. These models typically provide explicit second order integration in time and space of the conservation equations. Due to the extreme deformations often observed, Eulerian and ALE modelling are becoming commonly chosen methods over traditional Lagrangian approaches. As the requirement for increased resolution of urban wall constructions to accurately model munitions and wall structure responses constantly push existing computational platforms to their limits, methods to reduce computational time and memory requirements have proven to be extremely beneficial. In particular, a variety of mesh reduction methodologies have been successfully implemented and applied for use in the detailed modelling of the high velocity impact of urban wall constructions. These new mesh reduction techniques are proving successful in resolving high rate physical phenomena not previously successfully modelled. In previous modelling efforts of projectile impact against concrete walls, the steel reinforcement bars were not successfully resolved, due to the large memory requirements to resolve these relatively fine structures and the associated excessive run times. The steel reinforcement bars are an important part of the physical process, as they provide both significant strengthening for the wall and a higher density material within the concrete wall that can significantly damage the projectile during the concrete wall penetration process. Figure 1 presents previous modelling of concrete wall impact by a hardened munition configuration. The inclusion of steel reinforcement in the concrete wall could be an important aspect of the munition and urban wall response, but was not included due to the fine resolution mesh requirement that would have resulted in prohibitive memory and run times.
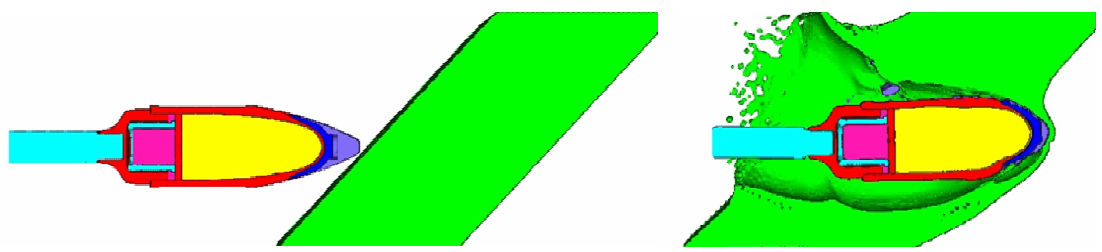

Figure 1: Previous modelling of concrete wall impact by a hardened munition configuration in which steel reinforcement bars were not included due to prohibitive memory and run time requirements $(0$ and $400 \mu \mathrm{s})$.

\section{Eulerian adaptive mesh refinement}

Adaptive mesh refinement (AMR) has been investigated as a method for improving computational resolution, reducing memory requirements and increasing computational efficiency for high rate continuum modelling by a number of researchers [1-3]. The implementation of such an adaptive mesh 
refinement capability has been recently completed by Sandia National Laboratories in the multi-materials high rate continuum computer model, CTH [4]. In order to achieve a practical implementation with good parallel performance, a block-based approach has been implemented with refinement and un-refinement occurring in an isotropic 2:1 manner. Crawford et al [5] showed that practical speed-up from 3 to 10 times and at least 3 times memory requirement reductions can be achieved on multiprocessor and massively parallel platforms. When a refinement indicator reaches its threshold, then an adjacent is signalled for refinement at least two cycles before the refinement is needed. This procedure has proven improved parallel efficiency, particularly when coupled with message passing consolidation and an efficient load balancing strategy. Currently implemented refinement indicators include velocity, pressure, temperature, materials and material boundaries as indicated by mixed cells.

We have applied this new capability to produce increased rectilinear mesh refinement in localized areas of interest for the detailed modelling of the high rate impact of urban structures. In particular, AMR has been used to successfully resolve geometry and physical phenomena involved in masonry wall impact modelling. Recent modelling efforts using AMR of concrete wall impact are now including steel reinforcement bars, which are successfully resolved. Figure 2 presents initial and late time plots of the projectile concrete wall penetration process. Figure 3 presents the initial state material region plot if the mesh refinement is not used. In this example, refinement indicators are used in order to refine everywhere individual volume fractions are greater than 0 (anywhere those materials exist), except for concrete. Three levels of refinement are used, with a minimum cell size of $1.6 \mathrm{~mm}$. In this way, the projectile and concrete reinforcement bars are highly refined, whereas the surrounding air (modelled as void) and concrete are less refined.

We have also applied this new capability to produce increased rectilinear mesh refinement in localized areas of interest for the detailed modelling of brick over concrete block masonry and triple brick masonry constructions. The AMR routines successfully resolve the detailed hollow cavity concrete block, brick and mortar geometries, as well as the physical phenomena involved in masonry wall impact. Figure 4 presents initial and late time plots of the projectile impact and penetration process against a brick over block masonry wall. Figure 5 presents initial and dynamic material plots of projectile impact against a triple brick masonry wall. As before, refinement indicators are used in order to refine individual volume fractions are greater than 0 (anywhere those materials exist), except for the brick material and three levels of refinement are used, with a minimum cell size of $1.6 \mathrm{~mm}$. In this way, the projectile, mortar and concrete block are highly refined, whereas the surrounding air (modelled as void) is less refined.

\section{Arbitrary Lagrange-Eulerian mesh relaxation}

In addition, a variety of mesh reduction relaxation algorithms have been developed for high rate continuum Arbitrary Lagrangian-Eulerian (ALE) 

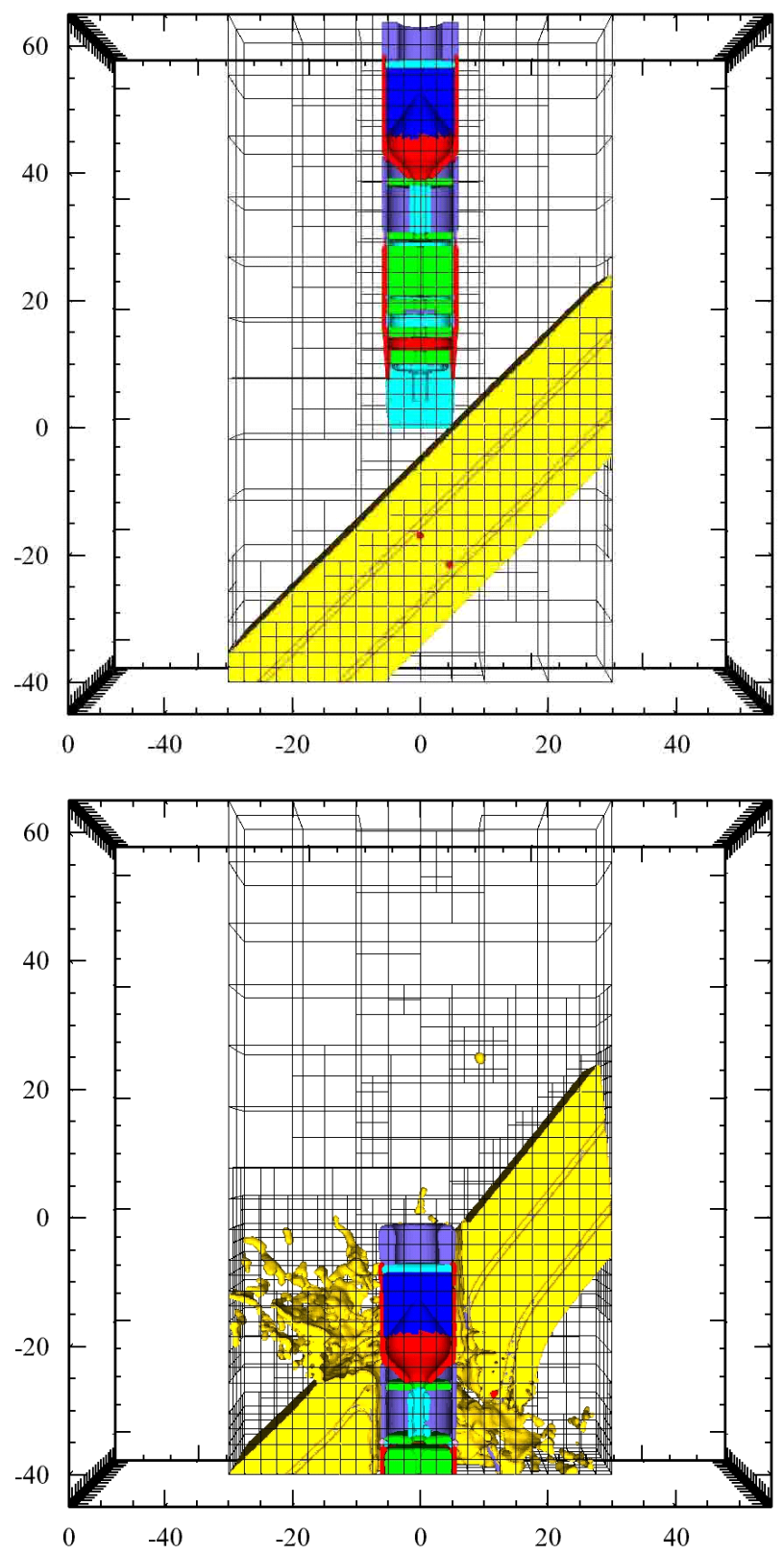

Figure 2: Initial and late time plots of the projectile concrete wall penetration process showing mesh blocks (dimensions in $\mathrm{cm}$ ). 

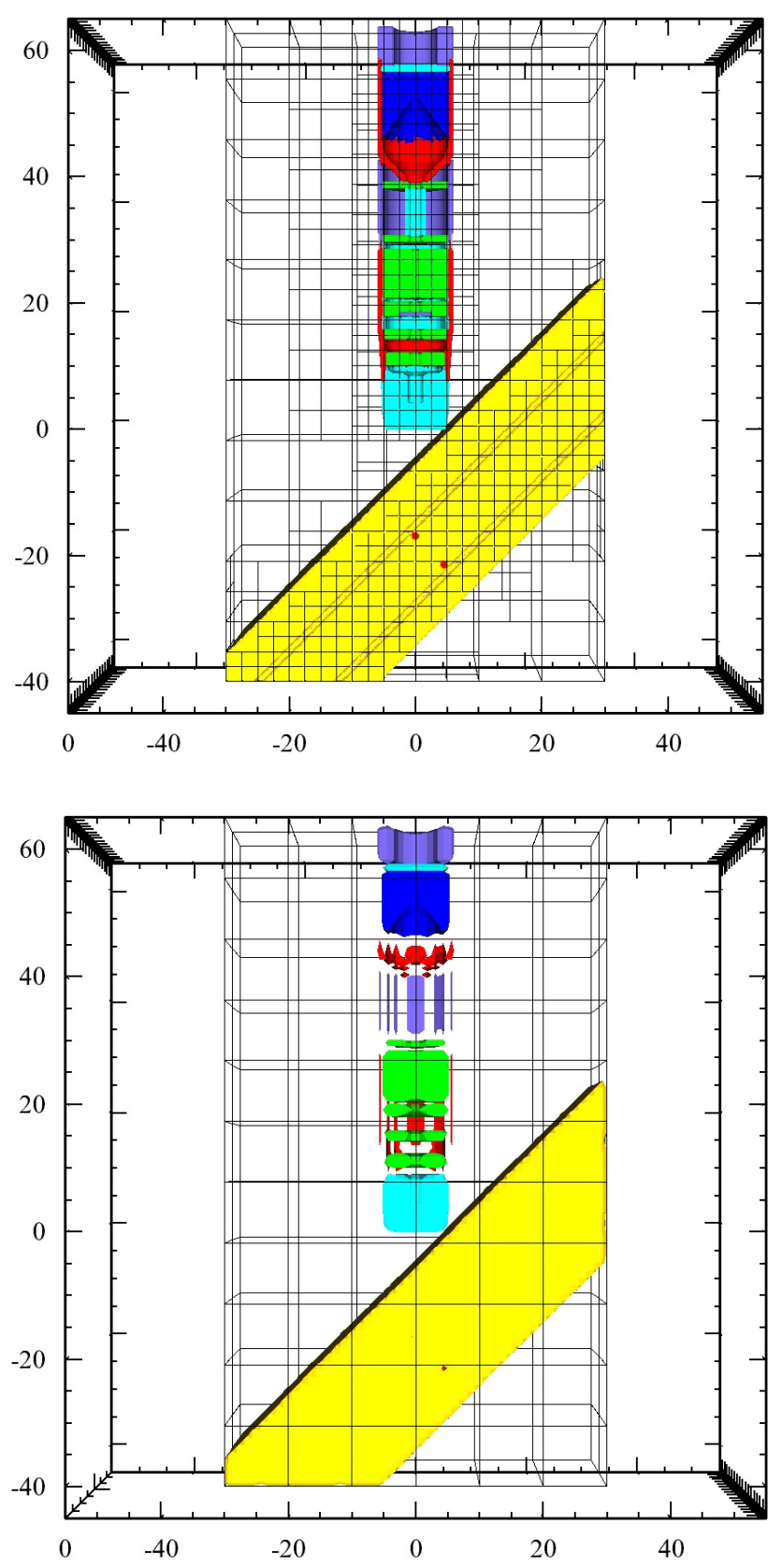

Figure 3: Initial state material region plot if the mesh refinement is not used. 

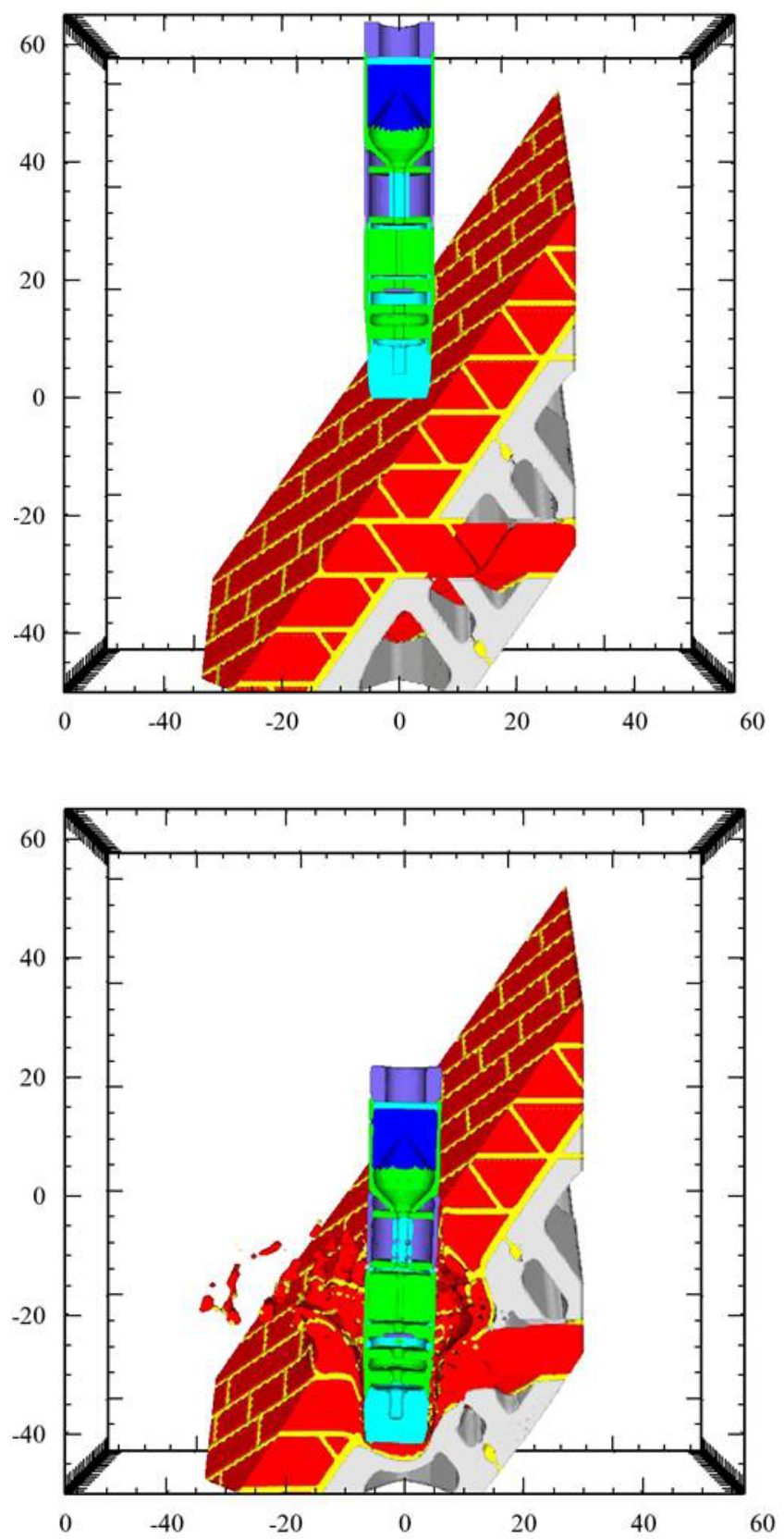

Figure 4: Initial and late time plots of the projectile brick over concrete block wall penetration process showing mesh blocks (dimensions in $\mathrm{cm}$ ). 

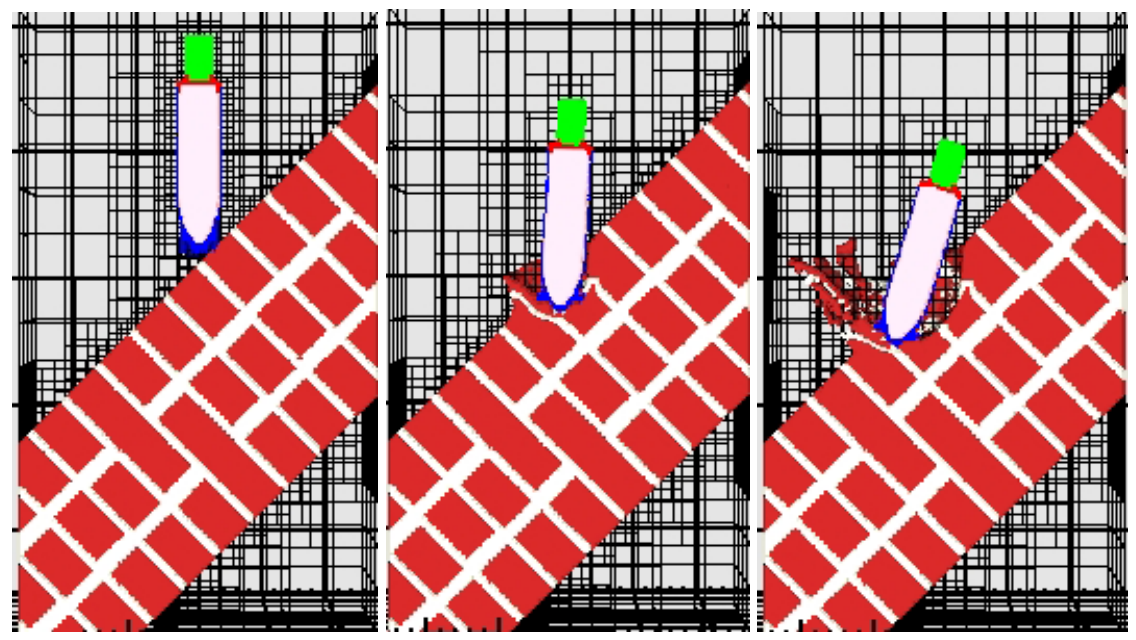

Figure 5: Initial and dynamic impact material plots of the projectile triple brick wall penetration process showing mesh blocks at $0,0.6 \mathrm{~ms}$ and $2.5 \mathrm{~ms}$.
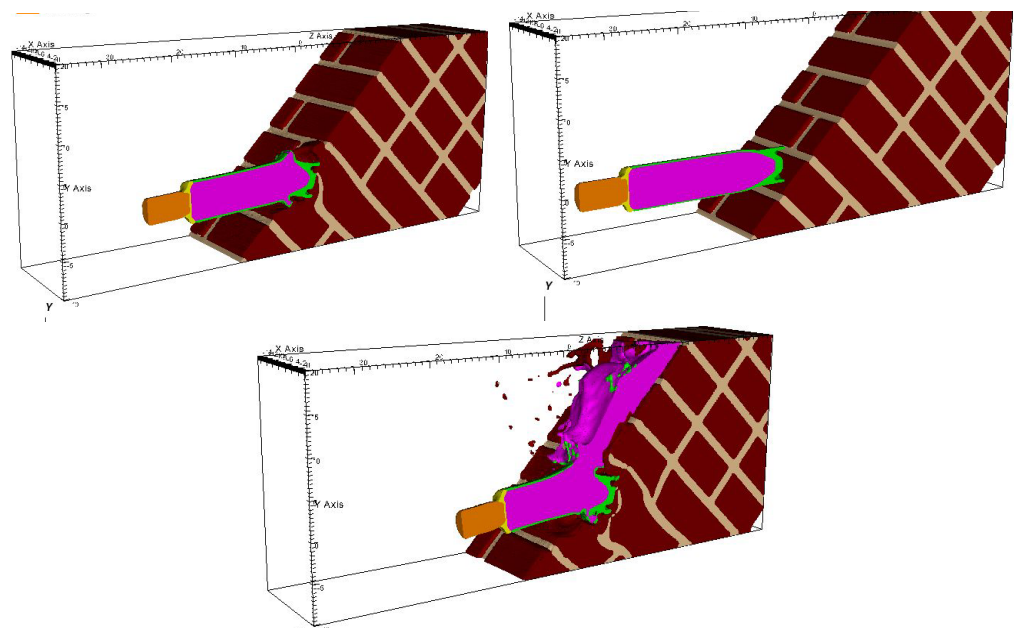

Figure 6: Material plots of the initial and dynamic wall impact ALE-3D simulation at $0,0.6 \mathrm{~ms}$ and $3.8 \mathrm{~ms}$.

modelling in the ALE-3D computer program [6-8]. These weighted relaxation algorithms have been successfully used to provide high resolution simulation of urban wall impact. For these simulations, material boundaries and materials are given higher weights, resulting in increased mesh refinement for associated localized regions. Typically, geometry will be initially input using a rectilinear 
mesh configuration. Before running, the weighted mesh relaxation algorithm is iterated, without integrating forward in time. In this way, the mesh will refine regions of interest before commencing the dynamic simulations. Figure 6 presents initial and late time material plots of urban wall projectile impact modelling using ALE-3D. Figure 7 presents a close view mesh plot for the initial mesh rectilinear and ALE relaxed configurations before the dynamic calculation and the dynamic ALE mesh during the impact simulation. A weighted potential algorithm [9] based on material fractions can be used in the ALE-3D program. In this way, the higher weighted materials result in increased resolution mesh areas. This can clearly been seen in the figures.

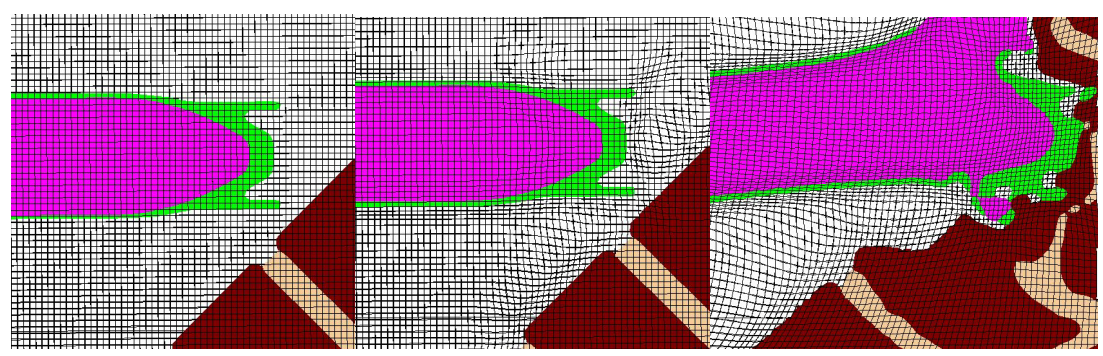

Figure 7: ALE-3D mesh plots: initial rectilinear mesh (left), initial relaxed ALE mesh (centre) and late time ALE mesh (right).

\section{Conclusions}

Recent advancements of continuum modelling are now allowing significant improvements of urban wall structures impact simulation. Urban wall structural details can now be included in impact modelling that were not previously achievable. These modelling improvements include the inclusion of resolved reinforcement bars in concrete walls, brick and mortar construction details in over block wall constructions. These modelling improvements are being achieved not only due in increases in computer hardware speeds and memory, but more so due to a variety of mesh reduction methodologies that are now being applied. The adaptive mesh refinement (AMR) technique implemented for use in the $\mathrm{CTH}$ high rate Eulerian finite difference model allows increased rectilinear mesh refinement in localized areas of interest. We have applied this AMR to successfully resolve complex material geometries and dominating physical phenomena involved in urban wall impact modelling. In addition, relaxation algorithms have been successfully applied for high rate continuum Arbitrary Lagrangian-Eulerian (ALE) modelling using ALE-3D. Munitions impacts at a variety of impact velocities and obliquities have now been successfully modelled against double reinforced concrete walls, brick walls and brick over hollow concrete blocks. These modelling techniques are now allowing the high rate continuum modelling of physical phenomena that was not previously simulated. 


\section{References}

[1] Berger, M.J. \& Colella, P., Local adaptive mesh refinement for shock hydrodynamics, J. Comp. Phys., 82, 64-84, 1989.

[2] Jones, B., SHAMROCK - an adaptive, multi-material hydrocode, Proc. of the International Workshop on new Models and Numerical Codes for Shock Wave Processes in Condensed Media, St. Catherines College, Oxford, UK, 15-19 September 1997.

[3] Tang, P.K \& Scannapieco, A.J., Modelling cylinder test, Proc. of the Conference of the American Physical Society Topical Group on Shock Compression of Condensed Matter, Seattle, WA, USA, August 13-18, 1995, 449-452.

[4] Crawford, D.A., Adaptive mesh refinement in CTH, Proc. of the 15th U.S. Army Symposium on Solid Mechanics, Myrtle Beach, South Carolina, USA, April 12-14, 1999.

[5] Crawford, D.A., Taylor, P.A., Bell, R.L. \& Hertel, E.S., Adaptive mesh refinement in the CTH shock physics hydrocode, Proc. of New Models and Hydrocodes for Shock Wave Processes in Condensed Matter, Edinburgh, U.K., May 19-24, 2002.

[6] Couch, R., Sharp, R., Otero, I., Neely, R., Futral, S., Dube, E., McCallen, R., Maltby, J. \& Nichols, A., ALE hydrocode development, Joint DOD/DOE Munitions Technology Development Progress Report, UCRL-ID-10348295, January 1996.

[7] Dube, E. \& Rodrigue, G., A geometric weighted elliptic grid regeneration method for 3D unstructured ALE hydrodynamics, Proc. of the 5th International Symposium on Computational Fluid Dynamics, Sendai, Japan, August 31-September 3, 1993.

[8] Baker, E.L., Pfau, D., Pincay, J.M., Vuong, T. \& Ng, K.W., High Rate Continuum Modeling Mesh Reduction Methodologies and Advanced Applications, Proc. of the 28th World Conference on Boundary Elements and Other Mesh Reduction Methods, Skiathos, Greece, 10-12 May 2006.

[9] Winslow, A. 1963, Equipotential zoning of two dimensional meshes, LLNL Report UCRL-7312, 1963. 